\title{
Evaluación de los aprendizajes sobre ciudadanía: meta evaluación de los instrumentos utilizados en el segundo ciclo básico chileno
}

\author{
Learning assessment on citizenship: meta-evaluation of the instruments \\ used in the Chilean second cycle of primary school
}

\author{
Karen Núñez Valdés \\ Pontificia Universidad Católica de Valparaíso \\ Telf.: (56) 322273385. Correo electrónico: karen.nunez@pucv.cl
}

\begin{abstract}
RESUMEN
Los diversos procesos que se han experimentado en los últimos tiempos, no sólo en nuestro país, sino que a nivel mundial, han derivado en diferentes discusiones en torno a la formación ciudadana. En el caso de Chile se ha hecho necesario incluir este eje temático en el currículum nacional, asumiéndose con ello la responsabilidad de educar a los estudiantes en temas ciudadanos. Este nuevo escenario ha planteado múltiples desafíos, especialmente en lo que se refiere a la evaluación de los conocimientos ciudadanos, por lo que en este estudio se pretende construir conocimiento, a partir del análisis de los instrumentos de evaluación con los que se evalúa la ciudadanía en el segundo ciclo básico. Para ello se ha planteado una metodología cualitativa, mediante la cual se analizan ítems de instrumentos tanto de tipo estandarizados como los diseñados por un grupo de docentes de la $\mathrm{V}$ región y que son aplicados en los cursos que componen este ciclo.
\end{abstract}

Palabras clave: formación ciudadana, evaluación en el aula, evaluación estandarizada.

\begin{abstract}
The different processes experienced in the last years, not only in our country but also internationally, have derived in different discussions around citizenship. In the Chilean case it has been necessary to include this central theme in the national curriculum; it is assumed then that there is a responsibility to educate students in citizenship topics. This new scenario has raised multiple challenges, especially regarding assessment of citizenship knowledge. In this study, we pretend to build knowledge from the analysis of different assessment tools used to assess students in the second cycle of primary school. In order to do this, a qualitative approach has been devised analyzing the items of the standardized instruments and those designed by a group of teachers of the 5th Region. They were applied in the classes that belong to this cycle.
\end{abstract}

Key words: citizen education, classroom assessment, standardized assessment. 


\section{INTRODUCCIÓN}

A partir de la década de los noventa el Estado chileno ha debido dar prioridad a la educación referida a la formación ciudadana, especialmente porque esta ha ocupado un lugar relevante en las diferentes discusiones que se han generado a nivel mundial, consecuencia de los diversos procesos que se han experimentado en los últimos tiempos: apatía de los votantes, creciente desigualdad, migraciones, globalización, entre otros, por lo que ha sido necesario incluir este eje temático en el currículum nacional, el cual es tratado en la actualidad en el sector de Historia, Geografía y Ciencias Sociales, asumiéndose con ello el desafío de educar en temas ciudadanos. Esta tarea no ha sido fácil, dado que la ciudadanía es un contenido vivido (Kymlicka \& Norman, 1997), que requiere de estrategias educativas y de una evaluación que permitan al estudiante poseer los conocimientos ciudadanos necesarios para desenvolverse en sociedad. Ello coloca en una encrucijada a los docentes quienes no solo deben enseñar este contenido, sino que también evaluarlo de forma coherente.

Por tal motivo esta investigación se ha planteado como objetivo evaluar los instrumentos de evaluación con los que se recoge información de los conocimientos ciudadanos de los estudiantes del segundo ciclo básico chileno: instrumentos estandarizados como la prueba SIMCE del área de Historia, Geografía y Ciencias Sociales, así como el diseño de instrumentos realizados por diferentes docentes de la $\mathrm{V}$ región y que son aplicados en los cursos que componen este ciclo en la asignatura de Historia, Geografía y Ciencias Sociales. Es relevante señalar que este estudio se ha realizado solo con los diseños de los instrumentos de evaluación, como consecuencia de las limitantes que surgieron en la recogida de estos.

El interés de este estudio se centra en conocer cómo se evalúa este eje temático vinculado a la ciudadanía, cuya importancia radica en el fin de todo proceso educativo, para dilucidar con ello para qué se educa a los sujetos, si para que estos aprendan a convivir en un espacio común, construyan conjuntamente este espacio o simplemente porque este contenido es parte obligatorio del currículum nacional. La evaluación de este contenido, tan importante para el desarrollo de nuestra sociedad permitirá conocer cómo los profesores miden este eje vinculado a la razón de ser, al sentido, a lo ético valórico de todo proceso educativo.

Esta investigación busca evaluar los instrumentos de evaluación que se aplican en el segundo ciclo básico chileno para el eje temático de formación ciudadana, con lo cual se persiguen los siguientes objetivos:

Objetivo 1: Identificar los contenidos referidos a la formación ciudadana que se evalúan en los instrumentos de evaluación diseñados para ser aplicados en el segundo ciclo básico.

Objetivo 2: Identificar las racionalidades curriculares que emergen de los instrumentos de evaluación aplicados en el segundo ciclo básico chileno.

Objetivo 3: Identificar las tipologías presentes en los instrumentos de evaluación utilizados en el segundo ciclo básico.

Objetivo 4: Analizar los modelos de evaluación que subyacen a las tipologías de evaluación.

En esta investigación se utiliza una metodología cualitativa, que involucra análisis del diseño de instrumentos de evaluación de un grupo de docentes de la $\mathrm{V}$ región y los ítems liberados de la prueba SIMCE, referidos al eje temático de formación ciudadana. 


\section{MARCO TEÓRICO}

El marco teórico que se presenta a continuación discute los conceptos que están sosteniendo una problemática de estudio vinculada a los instrumentos de evaluación con los cuales se emiten juicios referidos a los conocimientos ciudadanos de los y las niñas del segundo ciclo básico chileno.

\subsection{EL CONCEPTO DE CIUDADANÍA}

La ciudadanía se define y redefine necesariamente a partir de los contextos históricos, ya que cada grupo humano, a través de su historia y particularidades, establece una propia definición de esta, con su trasfondo teórico y filosófico. Esta característica tan propia del ser humano permite comprender la evolución que ha tenido el concepto y las aplicaciones que se le han dado en el transcurso de la historia occidental, especialmente en las últimas décadas, donde ha resurgido su discusión con gran fuerza como consecuencia de la crisis de las democracias representativas. La ciudadanía,

en su acepción clásica, se corresponde con un estatus jurídico y político mediante el cual el ciudadano adquiere, respecto a una colectividad política, unos derechos como individuo -civiles, políticos, sociales- y unos deberes impuestos. En su acepción moderna, se corresponde con el derecho y el deber de participación en la vida colectiva y democrática de un Estado. (Lozano, 2012, p. 1).

El concepto de ciudadanía como el de ciudadano data de hace unos 2.500 años atrás, pues es en Grecia, específicamente en Atenas, donde es posible ubicar su nacimiento. En los clásicos de la época se evidencia la importancia otorgada por los griegos a la convivencia y a la necesidad de normar esta, destacándose entre ellos la obra de Platón -"La República" (s. IV a. C./2010) y "Diálogos" (s. IV a. C./2009)- quien filosofó profundamente sobre esta temática. No obstante, es en la actualidad y en el contexto globalizado, donde el concepto adquiere gran preponderancia, pues las transformaciones sociales, políticas y económicas así lo han establecido, haciéndose evidente en el desarrollo de diversas teorías en torno al concepto de ciudadanía y por tanto del cómo convivir en sociedad (Habermas, 1998).

Es esencial identificar que la importancia del concepto radica en la naturaleza del ser humano, dado que somos seres sociales, Aristóteles ya lo planteaba en su obra la "Política" (s. IV a. C./2010), señalando además que necesitamos vivir en comunidad.

En el contexto latinoamericano actual, el concepto de ciudadanía no puede ser entendido sin otros, tales como el de consumo y medios de comunicación masivos, pues son estos los que hoy día marcan profundamente la cultura y la forma de convivir (Andrade \& Miranda, 2000). En la evolución histórica del concepto de ciudadanía se evidencia que el contexto delinea la forma de concebir y vivir esta en los diferentes períodos de la historia occidental, siendo relevante en ello los cambios y continuidades acaecidos para comprender el sentido actual del concepto y las dimensiones que ha adoptado este. 


\subsection{DIMENSIONES DEL CONCEPTO DE CIUDADANÍA}

En la actualidad, el concepto de ciudadanía va más allá de las consideraciones políticas y jurídicas que en algunas épocas fueron de gran relevancia, por ello para comprender la ciudadanía y sus teorías, como mínimo deben considerarse las siguientes dimensiones: política, social, económica, civil, intercultural, global o cosmopolita (Cortina, 2003). En este estudio se considerarán las dimensiones que a continuación se presentan.

\subsubsection{La ciudadanía desde una dimensión económica}

La sociedad en que hoy nuestros estudiantes se desenvuelven está teñida por los procesos de mundialización que se han venido experimentado a lo largo del siglo XX y "que se han acentuado por la aceleración de los intercambios comerciales entre naciones, puntualmente después de la firma, en 1947, del Acuerdo General sobre las tarifas aduaneras y el comercio" (Elbaz \& Helley, 2002, p. 46). Los intercambios comerciales, se han hecho cada vez más vertiginosos en la medida que las comunicaciones y el coste de estas se han reducido, multiplicándose con ello los flujos comerciales y financieros. Cada vez más se consumen productos extranjeros, aumentando con ello el comercio mundial. La mundialización es tanto más rápida cuanto que los flujos son cada vez menos materiales y conciernen cada vez más a los servicios, los datos informáticos, las telecomunicaciones, los mensajes audiovisuales, el correo electrónico, las consultas en Internet, etc. (Elbaz \& Helley, 2002). Este nuevo escenario plantea graves problemas de naturaleza política, pues se ha desarrollado una creciente complejización y diferenciación de las sociedades, tanto en el interior de sus relaciones como en las relaciones entre sociedades desarrolladas. El Estado perdió poder frente a la constitución de actores supranacionales y capacidad política a favor de los mercados abiertos, lo que resultó prácticamente imposible de regular (Calderón, 2007). Dada las características que han asumido los mercados, los Estados no han podido garantizar la equidad ni la inclusión social, desdibujando con ello grandes desafíos en lo que se refiere a la formación de ciudadanos en un mundo cada vez más globalizado.

La economía y la globalización de esta, han determinado que las capacidades políticas de los Estados nacionales se vinculen más con las oportunidades que se ofrecen en cuanto a las comunicaciones, a los recursos y a los intercambios comerciales, que a la búsqueda de igualdad y de inclusión social, problemas centrales en los gobiernos de ciertas regiones del mundo, América Latina es un ejemplo de esto. El Estado parece haberse colocado del lado de los mercados y alejado de la sociedad, pues debido a los procesos de globalización y al poder de los nuevos actores supranacionales, fueron perdiendo paulatinamente la capacidad de ejercer la soberanía nacional (Beck, 1998; Habermas, 1998; O’Donnell, 2006).

Si bien pareciera que la mundialización de la economía y la globalización de esta son oportunidades para los diferentes países en la integración del sistema mundial, las desigualdades cada vez se hacen más evidentes, pues la apertura de los mercados depende de las capacidades de los países, de las empresas y finalmente de las mismas personas. Esta situación queda de manifiesto cuando se comparan las capacidades de los países desarrollados con los países en vías de desarrollo, quienes deben limitarse por las decisiones de los primeros y que sin duda se ven afectados por lo que ocurre en estos (Calderón, 2007).

En lo referido al mundo laboral, este sufre las consecuencias de la flexibilización de los mercados, ya que la globalización no ha generado un mayor nivel de empleo ni la 
expansión de trabajos más calificados, por el contrario, los trabajadores hoy deben lidiar con la inestabilidad laboral, competir con los avances tecnológicos y afrontar la necesidad de una mayor capacitación. Los aspectos inequitativos de la globalización producen una gran exclusión socioeconómica, mayor pobreza y menores niveles de participación social y política. Como consecuencia, "se debilitan las condiciones de la ciudadanía y el mismo régimen democrático, que pierde legitimidad al no poder responderle de manera eficaz a la gente que se enfrenta a los diversos problemas asociados a la globalización económica" (Calderón, 2007, p. 36).

\subsubsection{La ciudadanía desde una dimensión político-jurídica}

A partir de la década de los setenta, los estados latinoamericanos abandonaron paulatinamente sus características de Estados de Bienestar para convertirse en Estados Subsidiarios, que dejan a merced de los privados ámbitos como la educación, la salud y la seguridad social. Los gobiernos de los países en vías de desarrollo tomaron medidas para insertarse en la economía global que afectaron significativamente a la mayoría de la población, pues disminuyeron su seguridad social por la reestructuración de la economía, especialmente a través de la privatización de empresas nacionales y de la liberalización de los mercados (Calderón, 2007). El Estado dejó de asegurar y gestionar el bienestar social al abandonar o disminuir sus responsabilidades sociales. Los Estados, con nuevas características, han debido continuar velando por la inclusión social y por la disminución de las desigualdades, cada vez con menos herramientas, ya que uno de los requerimientos de la globalización es precisamente que el Estado intervenga menos en la regulación de las economías nacionales, generándose con ello una tensión entre la economía y el rol del Estado.

En Chile, si bien la transición del régimen militar al democrático representó un sólido avance, este no se vio acompañado por un desarrollo político pleno, lo que es evidente en el debilitamiento de los partidos, expresado en el descenso de las afiliaciones y en el déficit del reclutamiento de personal político profesional (Cheresky, 2010). En Chile existe una legitimación de la democracia a partir del rendimiento económico, lo que concede un excesivo poder al empresariado y a los grupos corporativos fuertemente organizados, lo que acentúa la percepción de desigualdad en la población nacional (Cheresky, 2010). La situación antes descrita deviene de la historia propia de nuestro país en lo que se refiere a la ciudadanía, a su ejercicio y por tanto a su configuración. La Independencia de Chile constituyó todo un desafío para quienes debieron dar una nueva organización al país, existiendo desde este periodo una clara inclinación por gobiernos autoritarios y conservadores, lejos del ideario liberal que imperaba en la época. El Estado chileno del siglo XIX es poco más que un instrumento de la aristocracia, por lo que la misma idea de ciudadanía se encontraba subordinada al autoritarismo (Góngora, 1981).

Tras el fin de la Dictadura Militar, Chile entra en un nuevo proceso, donde es necesario recuperar las esferas propias de la ciudadanía, dado que los chilenos y chilenas se enfrentan a un nuevo escenario distinto al vivido por 17 años. Tal situación plantea al Estado la necesidad de reeducar a la población en los temas propios del convivir en sociedad. La mundialización, es decir, la conjunción de dos fenómenos: la intrincación cada vez más estrecha de las economías en el mercado mundial y las limitaciones o pérdida de poder de los Estados nacionales, representa un problema en lo que se refiere a la política (Elbaz \& 
Estudios Pedagógicos XLIII, Nº 2: 253-276, 2017

EVALUACIÓN DE LOS APRENDIZAJES SOBRE CIUDADANÍA: META EVALUACIÓN DE LOS INSTRUMENTOS UTILIZADOS EN EL SEGUNDO CICLO BÁSICO CHILENO

Helley, 2002). Esta disyuntiva descentraría la relación entre individuo y Estado que definía tradicionalmente la ciudadanía y pondría en primer plano la cuestión de la cultura como forma de pertenencia comunitaria sustitutiva.

\subsubsection{La ciudadanía desde una dimensión socio-cultural}

La globalización ha determinado grandes procesos de transformación en las relaciones no solo económicas y políticas, sino que también sociales y culturales, especialmente porque se ha dado inicio a una apertura de las fronteras, ya sea para intercambiar bienes materiales y/o servicios, como para la incorporación de preferencias e inclusive conductas. En esta transformación y específicamente en los aspectos socioculturales, el consumo se ha configurado como un eje de unificación y homogeneización de visiones, percepciones, motivaciones y comportamientos de las sociedades globales lo que lo convierte actualmente en un rasgo distintivo de estas (Fernández, 2013).

El consumo puede ser definido desde diversas ópticas, en cada una de las cuales se relevan diferentes racionalidades, las cuales intentan explicar el concepto y las relaciones que se generan a nivel social y cultural. El consumo es el conjunto de procesos socioculturales en que se realizan la apropiación y los usos de los productos, con lo cual se señala que el consumo no es un elemento divisor en las sociedades, sino que es parte de un código común, vale decir, posee un significado sociocultural, que se construye desde una racionalidad integrativa y comunicativa de la sociedad (García Canclini, 1991). El consumo también puede ser explicado desde una racionalidad sociopolítica interactiva, definido como un sitio donde los conflictos entre clases, originados por la desigual participación en la estructura productiva, se continúan a propósito de la distribución y apropiación de los bienes, es decir, se reconoce en el consumo la continuación de los conflictos de clases (Castells, 1974).

Dentro de las lógicas de una sociedad de consumo todo tiende a convertirse en mercancía, todo es potencialmente vendible y comprable. Además de este totalitarismo mercantil, el consumismo hunde sus raíces en valores directamente vinculados con un (ultra) individualismo, y promueve el interés individual y el ámbito privado por encima del bien común, de lo público y del interés colectivo (Fernández, 2013). Las sociedades consumistas carecen de dinámicas internas de responsabilidad moral respecto a los pobres y excluidos, pues revalorizan elementos propios de la lógica económica, los cuales determinan a un ciudadano cada vez menos responsable socialmente (Falk, 2002).

\subsection{LA ENSEÑANZA DE LA CIUDADANÍA Y EL CURRÍCULUM NACIONAL}

Tras la Dictadura Militar se iniciaron una serie de reformas en diferentes ámbitos, que pretendían posicionar a nuestro país en el nuevo contexto globalizado, pues no había existido una modernización acorde con este como consecuencia del contexto político imperante. Entre las reformas llevadas a cabo se destaca la Reforma Educacional (19961999), la que intentaba emprender cambios radicales a la educación con el fin de mejorar su calidad y hacerla más equitativa (Donoso, 2015). A principios de la década de los noventa la educación chilena estaba marcada por una fuerte escolarización, bajos resultados de aprendizajes y una mala distribución, lo que hizo que la agenda nacional en educación dejara de ser definida por el aumento del acceso a esta y pasara a centrarse en su calidad y equidad (García-Huidobro, 1999). 
En virtud de esta reforma se realiza un cambio en el currículo nacional. Estos cambios se enmarcan en el proceso de reincorporación de Chile al concierto internacional, del cual por casi 17 años de régimen militar había estado marginado. La experiencia política del país había generado una distancia entre el sistema político y la ciudadanía, el cual debía ser solucionado, a través de la política pública. En este contexto, la reforma curricular otorga gran importancia a la formación cívica, lo que es evidente en la misma definición del currículo, ya que busca entregar a los jóvenes las capacidades para desenvolverse con mayor propiedad en el ámbito personal, laboral y social; orientación que incluye naturalmente la formación de ciudadanos (García-Huidobro, 1999). En lo referido a la formación cívica, la reforma significó cambios importantes en los contenidos, pues antes de esta, existían asignaturas independientes que trataban el tema: "Educación Cívica", impartida en $4^{\circ}$ Medio y "Economía Política", dictada en $3^{\circ}$ Medio. "El foco de estas asignaturas, era el conocimiento del gobierno y sus instituciones" (Cox, Jaramillo \& Reimers, 2005, p. 19).

La reforma y el cambio curricular hicieron desaparecer ambas asignaturas para incluirse los contenidos de formación ciudadana transversalmente tanto en cursos de educación básica como de media, con lo cual se insertan en el área de Historia: Comprensión del Medio Natural Social y Cultural (NB1 y NB2), Estudio y Comprensión de la Sociedad (NB3 y NB4) y en Historia y Ciencias Sociales en educación media. Junto con este cambio curricular se establecen cambios en los contenidos; en el currículo pre reforma existía un énfasis en conceptos básicos de teoría política y de derecho, y en el conocimiento articulado de la Constitución política, donde además se trataban los temas de institucionalidad política y los derechos y deberes de los ciudadanos; en cuanto a la participación ciudadana, esta estaba restringida al sufragio. El cambio más importante en cuanto a los contenidos está ligado a que los conceptos de teoría política, institucionalidad, derechos y deberes del ciudadano, se vinculan con la realidad nacional, con la sociedad civil y con la inclusión de actividades de tipo social comunitaria, lo que se explica en gran medida porque detrás de estos cambios hay un giro en la concepción de educación cívica (García-Huidobro, 1999).

Avanzada la Reforma el año 2004, el Ministro de Educación de la época debió ir más allá, como consecuencia de las falencias que los estudiantes tenían en formación ciudadana, por lo que se decidió convocar a una comisión, llamada Comisión de Formación Ciudadana, cuya finalidad era

examinar los requerimientos formativos que plantea la ciudadanía democrática en las condiciones de la modernidad que Chile experimenta en los inicios de siglo, y como estos están siendo abordados por la educación, proponiendo mejoras o nuevos criterios y líneas de acción al respecto. (Comisión de Formación Ciudadana, 2004, p. 12).

Esta convocatoria surge por las características de nuestro país; la mayoritaria no inscripción en los registros electorales por parte de los jóvenes, la poca experiencia formativa en términos de participación ciudadana y el deber de la educación por formar a los y las ciudadanos del país.

Con el fin de graficar los principales cambios desarrollados a partir de la Reforma Educacional y de las iniciativas que desde la política pública surgieron en los últimos años, se presenta el Cuadro 1, donde se explicita el cambio de paradigma que experimentó el currículo nacional con la Reforma de fines de la década de los noventa: 
Estudios Pedagógicos XLIII, N²: 253-276, 2017

EVALUACIÓN DE LOS APRENDIZAJES SOBRE CIUDADANÍA: META EVALUACIÓN DE LOS INSTRUMENTOS UTILIZADOS EN EL SEGUNDO CICLO BÁSICO CHILENO

Cuadro 1. Cambio de paradigma, de educación cívica a educación ciudadana

\begin{tabular}{|l|l|}
\hline \multicolumn{1}{|c|}{ Educación Cívica } & \multicolumn{1}{c|}{ Formación Ciudadana } \\
\hline Foco en la institucionalidad política. & $\begin{array}{l}\text { Triple foco: institucionalidad política y aplicación } \\
\text { temática a "problemas actuales de la sociedad", y } \\
\text { a las competencias para resolver conflictos. }\end{array}$ \\
\hline $\begin{array}{l}\text { Ubicada en los últimos cursos de Educación } \\
\text { Media. }\end{array}$ & Presente a lo largo de toda la trayectoria escolar. \\
\hline $\begin{array}{l}\text { Orientada a la adquisición de conocimientos, } \\
\text { foco en contenidos. }\end{array}$ & $\begin{array}{l}\text { Orientada a la adquisición de conocimientos, } \\
\text { habilidades y actitudes en ambientes y prácticas } \\
\text { con predominio en relaciones participativas y } \\
\text { democráticas. }\end{array}$ \\
\hline
\end{tabular}

Fuente: Cox et al. (2005).

\subsection{EL CONCEPTO DE EVALUACIÓN EN EDUCACIÓN}

Siguiendo las propuestas actuales, la evaluación en el aula se entiende como un proceso complejo orientado a recoger evidencias respecto del aprendizaje de los estudiantes de manera sistemática, para emitir juicios en pos de un mejoramiento tanto de la calidad del aprendizaje como de la enseñanza. De acuerdo con esto se privilegia la función formativa de la evaluación, pues se le asocia con la mejora de los procesos de enseñanza y aprendizaje, dado que el profesor puede a través de esta hacerse consciente de la calidad de sus enseñanzas y los estudiantes por su parte pueden vislumbrar las falencias que poseen frente a sus procesos de aprendizajes, lo que eventualmente se traduce en el provecho de los recursos, del tiempo y de las potencialidades tanto de los profesores como de los estudiantes (Prieto \& Contreras, 2008). El proceso de evaluación adquiere así un papel relevante pues es el medio por el cual el profesor obtendrá información necesaria para ayudar a los alumnos a construir su conocimiento (Contreras, 2004).

\subsubsection{Evaluación comprensiva y evaluación basada en estándares}

Existen dos formas de enfocar la tarea evaluativa, las cuales no necesariamente deben ser entendidas como oponentes, sino que como dos caminos para realizar una evaluación, la que dependerá del contexto, de los estudiantes, de los docentes, entre otros elementos (Stake, 2006). Por una parte, está la evaluación orientada a las mediciones y, por otra, la orientada a la experiencia, por lo que existe la necesidad de que:

Los evaluadores conjuguen dos formas distintas de pensar y dos paradigmas de investigación que no forman, a priori, muy buena combinación. Se les suele denominar cualitativo y cuantitativo. Está bien llamarlos así. Yo he optado por referirme a uno de ellos como "comprensivo" o "interpretativo" y, al otro, como "basado en estándares", o "a partir de criterios. (Stake, 2006, p. 22).

a. Evaluación basada en estándares: Esta es definida "como un extenso grupo de 
métodos de evaluación que recurren principalmente al modo de pensar que se basa en criterios" (Stake, 2006, p. 107), estos pueden ser escritos o no, pero siempre son conscientes, vale decir se han concebido conjuntamente con la situación de evaluación, idealmente previa a esta. La evaluación basada en estándares supone un enfoque sumamente racional, dado ello mucho menos intuitivo en lo que se refiere a la percepción y emisión de un juicio en torno a la calidad de un determinado programa o tarea, pues se busca poseer un control en el desarrollo de la evaluación que permita asegurar la exactitud de los juicios a emitir.

b. Evaluación Comprensiva: Es una perspectiva más dentro de las formas de llevar a cabo una evaluación, pues puede permitir encontrar de otra manera la calidad y representación de un determinado programa, asignatura, contenido, etc. Para este autor:

Ser comprensivo o comprensiva significa guiarse por la experiencia de estar personalmente allí, sintiendo la actividad y la tensión, conociendo a las personas y sus valores. Es familiarizarse con las preocupaciones de los agentes implicados concediendo una atención adicional a la acción del programa, a su singularidad y a la pluralidad cultural de las personas. (Stake, 2006, p. 142).

Las evaluaciones de tipo comprensivas son más flexibles que otros enfoques de evaluación, tales como las evaluaciones basadas en estándares, dado que es en función de sus características que se puede adaptar en la medida que transcurra una unidad de aprendizaje, un programa, etc., lo que también significa que esta forma de evaluación dé menos pie a los datos cuantitativos y a la búsqueda de significaciones estadísticas, aumentando además el riesgo de que se implique emocionalmente el evaluador con ciertos grupos o posturas.

\subsubsection{Instrumentos de evaluación}

Un instrumento de evaluación es cualquier medio por el que se recoge la evidencia sobre el aprendizaje de los estudiantes, la cual, una vez analizada, permitirá emitir un juicio con fundamentos y tomar las mejores decisiones (Himmel, Olivares \& Zabalza, 1999). De acuerdo con esto, un instrumento de evaluación nos permite conocer qué tanto saben los estudiantes sobre un determinado contenido, asimismo nos permite reconocer el grado de avance de una determinada habilidad a desarrollar.

Los instrumentos de evaluación pueden adoptar diferentes formas, dado que obedecen a un propósito evaluativo que delineará no solo su formato sino que también los contenidos y habilidades que se desean evaluar. Atendiendo a esto es que existen diversas clasificaciones en torno a los tipos de instrumentos de evaluación. En la Tabla 1 se sintetizan los tipos de instrumentos de evaluación y sus características: 
Estudios Pedagógicos XLIII, N²: 253-276, 2017

EVALUACIÓN DE LOS APRENDIZAJES SOBRE CIUDADANÍA: META EVALUACIÓN DE LOS INSTRUMENTOS UTILIZADOS EN EL SEGUNDO CICLO BÁSICO CHILENO

Tabla 1. Tipos de instrumentos de evaluación y sus características

\begin{tabular}{|c|c|c|}
\hline Tipos de Instrumentos de Evaluación & Característica & Autores \\
\hline $\begin{array}{l}\text { Pruebas escritas de respuesta estructurada. } \\
\text { Pruebas de expresión escrita. } \\
\text { Pruebas estructuradas. } \\
\text { Pruebas semi-estructurada. } \\
\text { Evaluación del aprendizaje conceptual. }\end{array}$ & $\begin{array}{l}\text { Instrumentos de evaluación que se } \\
\text { han concebido bajo criterios de obje- } \\
\text { tividad. } \\
\text { Las respuestas a desarrollar son de } \\
\text { tipo cerradas, cuya característica } \\
\text { esencial es la reproducción de infor- } \\
\text { mación más que el desarrollo de ha- } \\
\text { bilidades superiores y, por tanto, de } \\
\text { aprendizajes profundos. }\end{array}$ & $\begin{array}{c}\text { Cortés (2009) } \\
\text { López (2009) } \\
\text { Carrasco (2004) } \\
\text { Giné (2000) }\end{array}$ \\
\hline $\begin{array}{l}\text { Instrumentos de evaluación basados en } \\
\text { ejecuciones. } \\
\text { Pruebas de expresión escrita. } \\
\text { Pruebas no estructuradas. } \\
\text { Evaluación de aprendizajes de procedi- } \\
\text { mientos. }\end{array}$ & $\begin{array}{l}\text { Instrumentos de evaluación basados } \\
\text { en preguntas con respuestas de tipo } \\
\text { abiertas y a modo de ensayo. } \\
\text { Se incorporan criterios de evaluación } \\
\text { más complejos (realización de un } \\
\text { procedimiento). }\end{array}$ & $\begin{array}{c}\text { Cortés (2009) } \\
\text { López (2009) } \\
\text { Carrasco (2004) } \\
\text { Giné (2000) }\end{array}$ \\
\hline $\begin{array}{l}\text { Evaluación de actitudes y personalidad. } \\
\text { Evaluación del aprendizaje de actitudes. }\end{array}$ & $\begin{array}{l}\text { Se caracterizan por la construcción } \\
\text { de encuestas y cuestionarios que per- } \\
\text { mitan analizar las actitudes de los } \\
\text { estudiantes. } \\
\text { Se releva la observación para evaluar } \\
\text { las actitudes de los participantes. }\end{array}$ & $\begin{array}{l}\text { Cortés (2009) } \\
\text { Giné (2000) }\end{array}$ \\
\hline
\end{tabular}

Fuente: elaboración propia.

\subsection{EVALUACIÓN DE LOS SABERES REFERIDOS A LA CIUDADANÍA}

La evaluación de los conocimientos y/o saberes de los estudiantes en torno a la formación ciudadana en nuestro país se realiza a través de diferentes procedimientos evaluativos, unos cuyo objetivo es más explícito que el de otros. En el caso de los estudiantes del segundo ciclo básico la evaluación de los conocimientos ciudadanos se realiza en dos modalidades:

a. A nivel de aula, a través de diferentes instrumentos de evaluación confeccionados por los y las docentes.

b. A nivel nacional, a través de pruebas de tipo estandarizadas tales como el SIMCE del área de Historia, Geografía y Ciencias Sociales, aplicada en Octavo básico.

\subsubsection{SIMCE sector Historia, Geografía y Ciencias Sociales}

En el caso de nuestro país, la evaluación en términos de políticas públicas y de asignación de recursos se ha focalizado en la aplicación de pruebas de tipo estandarizadas, cuya elección está fuertemente marcada por el contexto político nacional, dado que a partir de la década de los ochenta en Chile se inicia una progresiva transformación estatal, basada en 
la adopción de un nuevo sistema económico que propiciaba la liberación de la economía y junto con ello las cargas que el Estado debía asumir. Es así como el modelo neoliberal se impone en Chile, implicando ello la puesta en marcha de nuevas políticas educativas. En este nuevo contexto la educación comienza a ser traspasada progresivamente de manos del Estado a nuevos administradores, los cuales asumieron el rol que antaño le había correspondido a este. De este modo, las municipalidades y los inversionistas privados, a través de los colegios subvencionados y privados, son los encargados de proveer a la sociedad chilena de educación, con lo cual se acentúa el rol subsidiario del Estado, es decir, el Estado chileno entrega cuotas de dinero a estos nuevos administradores con el fin de que estos inviertan en la educación de los chilenos y chilenas (Maldonado, 2003). Esta necesidad se inserta en un contexto global en el que los Estados abandonan cada vez más su papel de proveedores directos de servicios para convertirse en supervisores del cumplimiento de las normas establecidas, regulando su actuación con evaluaciones de tipo estandarizadas (Ramírez, 2010). Este escenario creó la necesidad de monitorear la educación y por tanto la inversión que se estaba entregando, debido a lo cual surge un sistema de evaluación que permite conocer la calidad de la educación chilena. Este sistema se ha denominado SIMCE y ha intentado proveer al Estado de información sobre el manejo de la educación nacional y de la calidad de esta. Si bien sus propósitos se vinculan con el mejoramiento de la calidad y equidad de la educación, esto no se ha concretado. Las políticas educacionales chilenas poseen, una serie de puntos débiles en lo que se refiere a la equidad (OCDE, 2015).

El SIMCE pretende recabar información de los aprendizajes de los estudiantes en los distintos niveles de la enseñanza formal del país. Asimismo contempla recoger información sobre los docentes, los estudiantes y los padres y apoderados a través de cuestionarios de contexto, con el fin de contextualizar los resultados obtenidos por los estudiantes en las pruebas SIMCE. La prueba estandarizada SIMCE evalúa diferentes áreas del conocimiento, en el sector de Historia, Geografía y Ciencias Sociales se ha incorporado la evaluación tanto de los contenidos como de las habilidades referidas a la Ciudadanía, estos dependen del eje temático "Democracia y Desarrollo" (Agencia de la Calidad de la Educación, 2015).

\section{MARCO METODOLÓGICO}

En virtud de los objetivos propuestos se ha optado por una metodología de tipo cualitativa. La técnica a utilizar es el análisis de contenido, el que parte del supuesto de que el hablar y el escribir son también, por sí mismos, una forma de conducta social, pues lo que los hombres (genéricamente hablando) dicen o escriben revelan las intenciones, actitudes, interpretaciones, conocimientos y supuestos sobre su entorno (Ander-Egg, 1993). El análisis de contenido persigue como objetivo lograr la emergencia de aquel sentido latente que procede de las prácticas sociales y cognitivas que instrumentalmente recurren a la comunicación, de este modo el análisis de contenido pretende indagar en aquello que está oculto en el texto o bien profundizar en aquello que es evidente en este (Piñuel, 2002).

El objeto de análisis en esta investigación posee una base gramatical, ya que se analizarán las narrativas de las evaluaciones referidas al eje temático de formación ciudadana. En lo referido a la selección de las categorías analíticas, es importante destacar que cualquier análisis de contenido se sostiene o se hunde por cuáles sean sus categorías de análisis, 
por lo que la selección de estas siempre deben estar al alero de una teoría (Piñuel, 2002). Se distinguen cinco grupos de categorías: categorías de materia o contenido, de forma, de apreciación o de juicio, de personas y actores, y las de origen y de destino. En este estudio las categorías utilizadas son las de contenido, ya que se analizan las materias o contenidos presentes en los instrumentos de evaluación. El diseño del estudio es de tipo transversal, pues se analizan diferentes documentos (evaluaciones) sobre un mismo contenido (ciudadanía), los que han sido elaborados en un mismo tiempo histórico (Piñuel, 2002).

Se han definido dos grupos muestrales, por una parte, las evaluaciones diseñadas por docentes del área de Historia, Geografía y Ciencias Sociales para los cursos del segundo ciclo básico y, por otro lado, las preguntas liberadas por el MINEDUC de la prueba estandarizada SIMCE del área de Historia, Geografía y Ciencias Sociales aplicada en octavo básico. Se seleccionó como población a un grupo de 25 profesores que dictan la asignatura de Historia en los niveles del segundo ciclo básico, con la finalidad de que estos suministraran las pruebas que han diseñado y aplicado para el contenido de Ciudadanía en los cursos que imparten. La selección se realizó atendiendo a criterios de heterogeneidad: género, experiencia laboral y formación de postgrado. Del total de 25 profesores invitados, solo 10 aceptaron participar de esta investigación, quienes firmaron un Protocolo de consentimiento informado, donde se explicitan los objetivos del estudio, el alcance de este y la confidencialidad de los datos proporcionados. La muestra se conformó por 10 docentes: 6 mujeres y 4 hombres, con un promedio de experiencia laboral de 4.5 años, y con 4 docentes que tienen formación de postgrado.

El segundo grupo muestral corresponde a ítems de la prueba SIMCE del área de Historia, Geografía y Ciencias Sociales, específicamente los que se han liberado tras la aplicación de esta en diferentes años. El total de ítems analizados es de 13, los que corresponden a la aplicación de la prueba en diferentes años, a saber, 2004, 2011 y 2012.

Ambos grupos muestrales se analizaron descriptivamente en el programa Excel 2007, con el objetivo de identificar y catalogar la realidad empírica de los documentos, mediante la definición a priori de categorías. Las categorías se definieron en códigos, los que conformaron un libro de código. Los códigos aplicados se presentan en la Tabla 2. 
Tabla 2. Libro de Códigos

\begin{tabular}{|c|c|c|}
\hline Código 1 & Tipos de evaluación & $\begin{array}{l}\text { a. Evaluación basada en estándares. } \\
\text { b. Evaluación comprensiva. }\end{array}$ \\
\hline Código 2 & $\begin{array}{l}\text { Tipos de instrumentos de } \\
\text { evaluación }\end{array}$ & $\begin{array}{l}\text { a. Pruebas escritas de respuesta estructurada. } \\
\text { b. Pruebas escritas de respuesta semiestructurada o de } \\
\text { tipo cuestionario. }\end{array}$ \\
\hline Código 3 & Tipos de preguntas & $\begin{array}{l}\text { a. Preguntas que requieren de una respuesta larga. } \\
\text { b. Preguntas de respuesta corta. } \\
\text { c. Preguntas objetivas. } \\
\text { d. Otro tipo de preguntas (organizadores gráficos, } \\
\text { mapas, entre otros). }\end{array}$ \\
\hline Código 4 & Contenido a evaluar & $\begin{array}{l}\text { a. Historia del concepto de ciudadanía. } \\
\text { b. Dimensión económica del concepto de ciudadanía. } \\
\text { c. Dimensión político jurídica del concepto de } \\
\text { ciudadanía. } \\
\text { d. Dimensión socio cultural del concepto de ciudadanía. } \\
\text { e. Contenido socio antropológico. } \\
\text { f. Otros contenidos. }\end{array}$ \\
\hline Código 5 & Racionalidad curricular & $\begin{array}{l}\text { a. Racionalidad oficial centralizada. } \\
\text { b. Racionalidad o modelo de procesos. }\end{array}$ \\
\hline Código 6 & $\begin{array}{l}\text { Metacognición de los } \\
\text { contenidos de formación } \\
\text { ciudadana }\end{array}$ & $\begin{array}{l}\text { a. Análisis de casos. } \\
\text { b. Formulación de proyectos en la comunidad. }\end{array}$ \\
\hline
\end{tabular}

Fuente: elaboración propia.

\section{RESULTADOS}

Los resultados presentados responden a los objetivos propuestos para esta investigación y se presentan por cada nivel del segundo ciclo básico.

4.1. ANÁLISIS DE LAS EVALUACIONES DISEÑADAS POR DOCENTES DEL ÁREA DE HISTORIA, GEOGRAFÍA Y CIENCIAS SOCIALES PARA EL SEGUNDO CICLO BÁSICO

\section{Quinto básico}

El currículum que se ha definido para este nivel abarca contenidos de la Historia de Chile, iniciándose estos con los pueblos originarios hasta la conquista española. Además deben aprender la historia colonial chilena para finalizar con la independencia, sus causas, desarrollo y consecuencias de esta, en la formación del Estado Chileno (Ministerio de Educación, 2012). En términos de competencias ciudadanas los estudiantes deben reconocerse como ciudadanos, respetando la identidad de cada uno de los sujetos que componen la nación. El docente debe propiciar transversalmente que el estudiante sea capaz de vivir en sociedad, 
Estudios Pedagógicos XLIII, Nº 2: 253-276, 2017

EVALUACIÓN DE LOS APRENDIZAJES SOBRE CIUDADANÍA: META EVALUACIÓN DE LOS INSTRUMENTOS UTILIZADOS EN EL SEGUNDO CICLO BÁSICO CHILENO

respetando la diversidad que en ella existe (Ministerio de Educación 2012). Del análisis de las evaluaciones para quinto básico se desprenden los datos del Cuadro 2.

Cuadro 2. Resumen Análisis instrumentos Quinto básico

\begin{tabular}{|l|l|l|}
\hline \multicolumn{1}{|c|}{ Curso } & \multicolumn{2}{|c|}{ Quinto Básico } \\
\hline $\mathrm{N}^{\circ}$ de pruebas analizadas & 7 instrumentos \\
\hline $\mathrm{N}^{\circ}$ de ítems analizados & 100 \\
\hline Tipo de evaluación & Evaluación basada en estándares \\
\hline \multirow{4}{*}{ Tipo de pruebas } & Combinadas & 1 prueba \\
\cline { 2 - 3 } & Respuesta estructurada & 2 pruebas \\
\cline { 2 - 3 } & Cuestionario & 4 pruebas \\
\hline \multirow{4}{*}{ Tipo de preguntas } & Selección múltiple & $65 \%$ \\
\cline { 2 - 3 } & Pregunta de respuesta corta & $33 \%$ \\
\cline { 2 - 3 } & Organizador gráfico & $2 \%$ \\
\hline \multirow{4}{*}{ Contenidos evaluados } & Dimensión político-jurídica & $55 \%$ \\
\cline { 2 - 3 } & Dimensión socio-cultural & $20 \%$ \\
\cline { 2 - 3 } & Dimensión económica & $10 \%$ \\
\cline { 2 - 3 } & Otros contenidos & $13 \%$ \\
\cline { 2 - 3 } & Historia del concepto de ciudadanía & $2 \%$ \\
\hline \multirow{2}{*}{ Racionalidad curricular } & Racionalidad por objetivos \\
\hline \multirow{3}{*}{ Habilidades evaluadas } & Recordar & $71 \%$ \\
\cline { 2 - 3 } & Comprender & $28 \%$ \\
\cline { 2 - 3 } & Aplicar & $1 \%$ \\
\hline
\end{tabular}

Fuente: elaboración propia.

\section{Sexto básico}

En términos de formación ciudadana se espera que los estudiantes se reconozcan como ciudadanos y desarrollen una predisposición favorable hacia la vida en comunidad, en el marco de una sociedad democrática, poniendo en práctica los valores inherentes a ello. Se busca que reconozcan los ideales y las prácticas en las que se sustentan la ciudadanía y el Estado de derecho, y adquieran las herramientas necesarias para participar de forma activa, informada y responsable en la sociedad. Los estudiantes de sexto básico deberán aprender contenidos referidos al Estado, a la composición de este, a la Constitución política, a los derechos y deberes de los ciudadanos, a la participación ciudadana, todo ello en el marco de la Historia de Chile (Ministerio de Educación, 2012). Del proceso analítico a los instrumentos de evaluación de este nivel se obtuvieron los resultados del Cuadro 3. 
Cuadro 3. Resumen Análisis instrumentos Sexto básico

\begin{tabular}{|l|l|l|}
\hline \multicolumn{1}{|c|}{ Curso } & \multicolumn{2}{c|}{ Sexto Básico } \\
\hline $\mathrm{N}^{\circ}$ de pruebas analizadas & 17 instrumentos \\
\hline $\mathrm{N}^{\circ}$ de ítems analizados & 354 & 15 pruebas \\
\hline Tipo de evaluación & Evaluación basada en estándares \\
\hline \multirow{4}{*}{ Tipo de pruebas } & Combinadas & 2 pruebas \\
\cline { 2 - 3 } & Cuestionario & $81 \%$ \\
\hline \multirow{4}{*}{ Tipo de preguntas } & Preguntas objetivas & $16 \%$ \\
\cline { 2 - 3 } & Pregunta de respuesta corta & $2 \%$ \\
\cline { 2 - 3 } & Organizador Gráfico & $77 \%$ \\
\hline \multirow{4}{*}{ Contenidos evaluados } & Dimensión político-jurídica & $7 \%$ \\
\cline { 2 - 3 } & Dimensión socio-cultural & $6 \%$ \\
\cline { 2 - 3 } & Dimensión económica & $4 \%$ \\
\cline { 2 - 3 } & Otros contenidos & $79 \%$ \\
\cline { 2 - 3 } & Historia del concepto de ciudadanía & $4 \%$ \\
\hline Racionalidad curricular & Racionalidad por objetivos \\
\hline \multirow{4}{*}{ Habilidades evaluadas } & Recordar & $18 \%$ \\
\cline { 2 - 3 } & Comprender & $3 \%$ \\
\cline { 2 - 3 } & Aplicar & \\
\hline
\end{tabular}

Fuente: elaboración propia.

\section{Séptimo básico}

Para el nivel de séptimo básico se han definido como habilidades a evaluar en el ámbito de la formación ciudadana la participación en una sociedad plural y la comunicación y valoración de los derechos y deberes. Estas deben ser trabajadas transversalmente en los diferentes contenidos, los cuales se vinculan con la historia universal (Ministerio de Educación, 2013). De los instrumentos analizados para este nivel se desprenden los datos del Cuadro 4. 
Estudios Pedagógicos XLIII, N²: 253-276, 2017

EVALUACIÓN DE LOS APRENDIZAJES SOBRE CIUDADANÍA: META EVALUACIÓN DE LOS INSTRUMENTOS UTILIZADOS EN EL SEGUNDO CICLO BÁSICO CHILENO

Cuadro 4. Resumen Análisis instrumentos Séptimo básico

\begin{tabular}{|l|l|l|}
\hline \multicolumn{1}{|c|}{ Curso } & \multicolumn{2}{c|}{ Séptimo Básico } \\
\hline $\mathrm{N}^{\circ}$ de pruebas analizadas & 23 instrumentos \\
\hline $\mathrm{N}^{\circ}$ de ítems analizados & 553 \\
\hline Tipo de evaluación & Evaluación basada en estándares \\
\hline \multirow{4}{*}{ Tipo de pruebas } & Combinadas & 100 pruebas \\
\hline \multirow{5}{*}{ Tipo de preguntas } & Preguntas objetivas & $69 \%$ \\
\cline { 2 - 3 } & Pregunta de respuesta corta & $24 \%$ \\
\cline { 2 - 3 } & Organizador gráfico & $2 \%$ \\
\cline { 2 - 3 } & Topónimos & $5 \%$ \\
\hline \multirow{5}{*}{ Habilenidos evaluados } & Dimensión político-jurídica & $36 \%$ \\
\cline { 2 - 3 } & Dimensión socio-cultural & $9 \%$ \\
\cline { 2 - 3 } & Otros contenidos & $47 \%$ \\
\cline { 2 - 3 } & Historia del concepto de ciudadanía & $8 \%$ \\
\hline Racionalidad curricular & Racionalidad por objetivos \\
\hline \multirow{5}{*}{ Habadas } & Recordar & $75 \%$ \\
\cline { 2 - 3 } & Comprender & $22 \%$ \\
\cline { 2 - 3 } & Aplicar & $3 \%$ \\
\hline
\end{tabular}

Fuente: elaboración propia.

\section{Octavo básico}

Considerado el curso terminal de la enseñanza básica de los estudiantes chilenos, y donde se aplica el SIMCE de Historia, Geografía y Ciencias Sociales, el Octavo Básico se constituye como la antesala a la formación que recibirán los estudiantes en la educación media. Corresponde por tanto a una síntesis de lo aprendido durante la enseñanza básica en lo referido a la formación ciudadana. Del proceso analítico a los instrumentos de evaluación de este nivel se obtuvieron los resultados presentados en el Cuadro 5. 
Cuadro 5. Resumen Análisis instrumentos Octavo básico

\begin{tabular}{|c|c|c|}
\hline Curso & \multicolumn{2}{|c|}{ Octavo Básico } \\
\hline $\mathrm{N}^{\circ}$ de pruebas analizadas & \multicolumn{2}{|l|}{9 instrumentos } \\
\hline $\mathrm{N}^{\circ}$ de ítems analizados & \multicolumn{2}{|l|}{197} \\
\hline Tipo de evaluación & \multicolumn{2}{|c|}{ Evaluación basada en estándares } \\
\hline \multirow{3}{*}{ Tipo de pruebas } & Combinadas & 7 pruebas \\
\hline & Respuesta estructurada & 1 prueba \\
\hline & Ensayo & 1 prueba \\
\hline \multirow{4}{*}{ Tipo de preguntas } & Preguntas objetivas & $77 \%$ \\
\hline & Pregunta de respuesta corta & $21 \%$ \\
\hline & Organizador gráfico & $1.6 \%$ \\
\hline & Pregunta de respuesta larga & $0.4 \%$ \\
\hline \multirow{3}{*}{ Contenidos evaluados } & Dimensión político-jurídica & $71 \%$ \\
\hline & Dimensión socio-cultural & $6 \%$ \\
\hline & Otros contenidos & $23 \%$ \\
\hline Racionalidad curricular & \multicolumn{2}{|l|}{ Racionalidad por objetivos } \\
\hline \multirow{4}{*}{ Habilidades evaluadas } & Recordar & $78 \%$ \\
\hline & Comprender & $20 \%$ \\
\hline & Aplicar & $1 \%$ \\
\hline & Analizar & $1 \%$ \\
\hline
\end{tabular}

Fuente: elaboración propia.

4.2. ANÁLISIS DE ÍTEMS LIBERADOS DE LA PRUEBA ESTANDARIZADA SIMCE DE HISTORIA, GEOGRAFÍA Y CIENCIAS SOCIALES - OCTAVO BÁSICO: EJE TEMÁTICO FORMACIÓN CIUDADANA

Resulta relevante señalar que la recopilación de ítems de la prueba SIMCE no es una tarea fácil, dado que anualmente se libera un pequeño grupo de estos, los que se colocan a disposición de los investigadores, profesores, directivos y la opinión pública. Asimismo, en el caso del SIMCE de Historia, Geografía y Ciencias Sociales, no todas las preguntas evalúan contenidos asociados a la formación ciudadana (25\% del total de los ítems de la prueba), por tanto, existen más dificultades a la hora de buscar este tipo de ítems. Es por esta razón que la muestra en términos de la prueba SIMCE se remite a ítems y no al análisis de una prueba íntegra.

Como primer elemento a considerar mencionaremos que los ítems que componen la prueba SIMCE se encuentran basados en estándares, vale decir existe un criterio pre existente que en este caso es establecido por el MINEDUC. A continuación, en el Cuadro 6, se presentan los análisis realizados a los ítems del SIMCE de Historia: 


\begin{tabular}{|l|l|l|}
\hline \multicolumn{3}{|l}{ Ítems SIMCE Octavo Básico área de Historia, Geografía y Ciencias Sociales } \\
\hline $\mathrm{N}^{\circ}$ de ítems analizados & 13 & \multicolumn{2}{|l|}{} \\
\hline Tipo de evaluación & Evaluación basada en estándares \\
\hline Tipo de pruebas & Combinadas & \multicolumn{2}{|l}{} \\
\hline \multirow{2}{*}{ Tipo de preguntas } & Preguntas objetivas & 1 preguntas \\
\cline { 2 - 3 } & Pregunta de respuesta abierta \\
\hline \multirow{4}{*}{ Contenidos evaluados } & Dimensión político-jurídica & 6 preguntas \\
\cline { 2 - 3 } & Dimensión económica & 1 pregunta \\
\cline { 2 - 3 } & Historia del concepto de ciudadanía & 6 preguntas \\
\hline Racionalidad curricular & Racionalidad por objetivos & \\
\hline \multirow{2}{*}{ Habilidades evaluadas } & Recordar & 11 preguntas \\
\cline { 2 - 3 } & Comprender & 2 preguntas \\
\hline \multirow{2}{*}{ Metacognición } & Sí & 4 preguntas \\
\cline { 2 - 3 } & No & 9 preguntas \\
\hline
\end{tabular}

Fuente: elaboración propia.

\section{DISCUSIÓN}

Bien es sabido que la enseñanza de la ciudadanía está compuesta por diferentes contenidos, los cuales están vinculados no solo a su surgimiento e itinerario histórico, sino que también a sus dimensiones, alcances e importancia, los que en su conjunto permiten a los sujetos acercarse a lo que se comprende por ciudadanía. Se entiende, como consecuencia de esto, que todos los contenidos que componen el eje temático de formación ciudadana poseen relevancia a la hora de formar ciudadanos, pues cada uno de ellos contribuye al conocimiento necesario para esta tarea. Entonces, nos situamos en la lógica de que los contenidos antes mencionados son esenciales a la hora de enseñar este eje temático tan sustantivo a todo proceso educativo, pues contribuirá a una formación integral de los estudiantes, por lo que a priori se espera encontrar evaluaciones que posean todo tipo de contenidos referidos a la formación ciudadana, especialmente porque el Estado chileno ha declarado que tras la Reforma Educacional de los años noventa, se ha transitado desde un currículum de educación cívica a uno de formación para la ciudadana (Cox et al., 2005).

La educación cívica se caracterizaba porque se trataban las temáticas ciudadanas en los últimos cursos de la enseñanza media, siendo el foco la institucionalidad política orientada a la adquisición de conocimientos. El nuevo enfoque, por su parte, posee un triple foco: institucionalidad política y aplicación temática a "problemas actuales de la sociedad", y a las competencias para resolver conflictos, con lo cual se integra la temática a lo largo de toda la trayectoria escolar, con la finalidad de que los estudiantes adquieran conocimientos, 
habilidades y actitudes en ambientes y prácticas con predominio en relaciones participativas y democráticas. De acuerdo a lo anterior, se deduce que el currículum actual crea las instancias necesarias para enseñar la ciudadanía desde diferentes contenidos y estrategias, dado que los nuevos lineamientos planteados por el Estado llaman a los docentes a enfocar su tarea en la formación de los futuros ciudadanos, con los suficientes conocimientos para vivir en sociedad. Sin embargo, lo acontecido en las aulas escolares, en términos de la evaluación de la ciudadanía, deja en evidencia las tensiones que existen en torno al cambio de paradigma, pues no solo encontramos instrumentos evaluativos enfocados más en la reproducción de conocimientos que en el desarrollo de habilidades, sino que también los contenidos que se evalúan en el segundo ciclo básico son esencialmente contenidos asociados a la dimensión político-jurídica de la ciudadanía, vale decir, se le otorga gran relevancia a esta dimensión por sobre las otras dimensiones que componen la temática. En este sentido, existe una reducción de los contenidos ciudadanos que se evalúan, pues estos se circunscriben en una sola dimensión, y se abandonan otras aristas igual de importantes que los elementos políticos que deben conocer los estudiantes. Esto nos hace reflexionar sobre lo que se enseña en las aulas escolares chilenas, pues lo que se evalúa es lo que enseña, por tanto, se infiere que existe un énfasis en la enseñanza de la ciudadanía desde sus elementos político-jurídicos y que se deja de lado la mirada socio- antropológica de esta. Esta situación puede ser explicada por diferentes elementos, entre los que según el análisis presentado destacan que el SIMCE posee un énfasis en la evaluación de esta dimensión, lo que posee una influencia en el diseño de las evaluaciones aplicadas en el aula, cuya característica esencial son las preguntas objetivas referidas a contenidos sobre la institucionalidad política.

Los contenidos identificados están íntimamente relacionados con la institucionalidad política, lo que se encuentra más asociado al paradigma de educación cívica que al de formación ciudadana actualmente planteado. De este modo se espera que los estudiantes comprendan la ciudadanía más desde sus elementos políticos que desde su trascendencia en el desarrollo de la sociedad; a la representación política, específicamente a los partidos políticos, a su definición y características; la historia del sufragio y los logros alcanzados por los diferentes grupos sociales en torno a este, los poderes del Estado, la definición de cada uno de estos, sus atribuciones y sus representantes, para finalmente encontrarnos con ítems referidos a los sistemas de gobierno y a su evolución política en la historia universal. Debemos recordar que el referente ha sido occidente, lo que es inalterable en las aulas chilenas debido a la herencia de la cultura occidental, con un mayor énfasis en el conocimiento de los contextos internacionales por sobre los nacionales, vale decir los estudiantes conocen con mayor profundidad lo ocurrido en Grecia que en nuestro propio país.

Las dimensiones económica y socio-cultural han sido evaluadas en menor medida en los instrumentos analizados, por lo que las preguntas referidas a estas se presentan de forma muy acotada y se refieren más a las características económicas de una determinada época de estudio que a los elementos que influyen o influyeron en la conformación de la ciudadanía. Resulta llamativo que no existan preguntas asociadas a la dimensión económica que evalúen elementos tales como la mundialización de la economía (Elbaz \& Helley, 2002) considerando que esta ha tenido un gran impacto no solo en el devenir económico de los estados sino que en la definición de estos, fenómeno que ha afectado las relaciones sociales de los distintos países y las relaciones entre Estados, pues el grado de participación en el concierto internacional ha comenzado a perfilar el tipo de relaciones que se establecen 
en sociedad. Otro elemento que es invisible en las evaluaciones es lo que Castells (1996) ha denominado sociedad red, la cual se caracteriza por el aumento del capital con respecto al trabajo, lo que implica un cambio profundo en las relaciones laborales, la regulación de los mercados y en la flexibilización laboral (Calderón, 2007), contenidos que permitirían eventualmente a los estudiantes analizar y reflexionar sobre la situación actual, donde la desigualdad, el desempleo y la inequidad han aumentado considerablemente. Sin el conocimiento de estos contenidos se hace dificultoso lograr habilidades críticas en los pequeños ciudadanos, pues carecen de elementos teóricos para llevar a cabo esta tarea.

Por su parte, la dimensión socio-cultural es la menos evaluada, lo que es evidente por la escasez de preguntas asociadas a esta. En los instrumentos analizados existen ítems que se refieren a elementos culturales característicos de una determinada época, y no se evidencian ítems sobre conceptos como consumo, el que ha sido trabajado por García Canclini (1991), Bourdieu (1998), Castells (1974) ni tampoco el de individualismo (Fernández, 2013), lo que puede ser explicado eventualmente porque las unidades a trabajar en el segundo ciclo básico están referidas a épocas precedentes, lo que hace más compleja su incorporación, considerando que los estudiantes no superan los 14 años, de igual modo el currículum del sector de Historia posee una gran cantidad de contenidos, lo que dificulta aún más la inclusión de estos conceptos. Asimismo, se han dejado de lado temas asociados a la importancia que se le otorga a los elementos propios de la lógica económica en la actualidad (Falk, 2002), por lo que no existe la posibilidad de que los estudiantes realicen una reflexión en torno a las dinámicas sociales actuales y a la influencia que puedan tener estas en la construcción del ciudadano.

En lo que se refiere a la Historia de la ciudadanía, se evalúan las bases de esta desde una visión histórica, en la cual indiscutiblemente el caso de Atenas posee mayor preponderancia. Si bien se valoriza el legado greco, existen vacíos en términos temporales, pues los estudiantes deben relacionar el surgimiento de la democracia en Grecia con las democracias actuales, aun cuando son contextos diametralmente distintos.

En términos de la racionalidad curricular, se evidencia que nuestro país posee un currículum por objetivos, también denominado racionalidad oficial centralizada (Stenhouse, 2003), dado que la administración, vale decir el Estado, a través del Ministerio de Educación decide la cultura básica a transmitir, decisión que es comunicada a los docentes a través de los diversos documentos emanados por esta institución, lo que incluye evidentemente las pruebas de tipo estandarizadas que se aplican en Chile. El tipo de modelo curricular que se posee en la actualidad dificulta la tarea de enseñar la ciudadanía como un conocimiento vivido, en el cual la evaluación puede ser adaptada según el contenido, el contexto, las características de los estudiantes, etc., ello porque debe darse cumplimiento a los estándares que se han planteado a nivel central. Como ya hemos señalado, los instrumentos de evaluación poseen características propias de la prueba estandarizada SIMCE, pues se evalúa preferentemente a través de preguntas objetivas, tales como selección múltiple, verdadero o falso, términos pareados, entre otras, con lo cual se renuncia a la posibilidad de que el eje de formación ciudadana sea una palanca de transformación social, donde el docente pueda decidir qué enseñar y cómo evaluarlo para que sus estudiantes aprehendan este contenido. La medición de los conocimientos de los estudiantes está dada por las diferentes habilidades que estos deben desarrollar, siendo un referente la taxonomía de Bloom en sus versiones revisadas, por lo que no es de extrañar que los instrumentos posean como objetivos de aprendizajes los que se han señalado en estas. A nivel del segundo 
ciclo básico predominan habilidades de aprendizaje tales como recordar y comprender, sin evidencia de otras que son consideradas de nivel superior como evaluar y crear.

Los instrumentos de evaluación utilizados en los diferentes cursos del segundo ciclo básico se caracterizan por ser en su totalidad evaluaciones basadas en estándares, con preponderancia de las pruebas escritas de tipo combinadas. De este modo en quinto básico mayoritariamente las pruebas son escritas de tipo cuestionario, con preguntas cuya habilidad es "recordar", vale decir, reproducir información, pues el estudiante debe limitarse a identificar y a relacionar conceptos, lo que sin duda no permite que la ciudadanía sea entendida como un conocimiento vivido. En términos generales las evaluaciones diseñadas para este nivel pretenden evaluar contenidos conceptuales, sin apreciarse otro tipo de contenidos tales como procedimentales y actitudinales, por lo que el desarrollo de habilidades para la vida ciudadana resulta muy difícil de lograr bajo situaciones de evaluación de este tipo, dado que estas no están enfocadas en evaluar las competencias ciudadanas. En sexto y en séptimo básico observamos que el tipo de prueba aplicada es esencialmente la prueba escrita de tipo combinada, donde existe una predominancia de las preguntas de tipo objetivas, lo que dificulta irremediablemente la evaluación de un conocimiento vivido que debe ser conectado con la realidad. Si bien existe un esfuerzo por diseñar preguntas que contextualicen al estudiante no se logra este propósito, dado que solo se espera que este comprenda lo que es la ciudadanía. Cabe destacar que se evidencian incoherencias entre lo que se declara estar evaluando con aquello que realmente se evalúa, pues existen objetivos de aprendizaje que enuncian el "análisis" cuando realmente los estudiantes solo deben "identificar". El panorama no es muy diferente en octavo básico, dado que se mantienen las mismas dinámicas de los cursos precedentes, es decir, mayoritariamente se evalúa a través de pruebas escritas de tipo combinadas, donde el estudiante debe recordar y comprender conceptos asociados a la formación ciudadana. En consecuencia, a lo largo del segundo ciclo básico nos encontramos con evaluaciones que se enmarcan en una lógica de educación cívica, donde lo más importante es lograr el conocimiento de un determinado contenido y no su aplicación en contextos reales. Este tipo de evaluación se relaciona directamente con el formato y contenido de la prueba SIMCE en el sector de Historia, Geografía y Ciencias Sociales, dado que esta es una evaluación de tipo estandarizada, donde las preguntas son esencialmente de selección múltiple, además de un pequeño grupo de preguntas de respuesta abierta. Esta situación fue narrada por los docentes, quienes reconocían que en su ejercicio profesional se les solicitaba la incorporación de preguntas tipo SIMCE para habituar a los estudiantes a esta prueba, lo que coloca en evidencia cómo las lógicas del mercado influyen poderosamente en la enseñanza y en la evaluación, pues obtener un alto puntaje en el SIMCE no solo significa prestigio a nivel social, sino que también incentivos económicos (excelencia académica por ejemplo, la cual se obtiene tras la conjugación de diferentes otros elementos), pues no debemos olvidar que nuestro sistema educacional es heterogéneo, siendo el rol del Estado netamente subsidiario. Tanto las evaluaciones diseñadas por los docentes como el SIMCE, dependiente del Estado chileno, se encuentran distantes de lo que ha planteado la comunidad internacional en términos de formación ciudadana, ya que en las pruebas aplicadas internacionalmente no existen respuestas correctas o incorrectas, ya que el objetivo principal de estas es conocer las preferencias de los estudiantes en un determinado contexto.

En nuestro país predomina como modelo de evaluación, la evaluación basada en estándares, la cual se caracteriza por poseer una serie de criterios de evaluación. Este 
Estudios Pedagógicos XLIII, Nº 2: 253-276, 2017

EVALUACIÓN DE LOS APRENDIZAJES SOBRE CIUDADANÍA: META EVALUACIÓN DE LOS INSTRUMENTOS UTILIZADOS EN EL SEGUNDO CICLO BÁSICO CHILENO

modelo supone un enfoque sumamente racional, por ello mucho menos intuitivo en lo que se refiere a la percepción, ya que se busca poseer un control en el desarrollo de la evaluación que permita asegurar la exactitud de los juicios a emitir. Se opta mayoritariamente por este modelo con el fin de controlar la subjetividad y el sesgo, operacionalizando los factores de las evaluaciones. Estas características que hacen de la evaluación basada en estándares un modelo ampliamente utilizado en diversas áreas son discutibles a la hora de evaluar contenidos como los de formación ciudadana, pues este no es cualquier contenido. En este sentido existe una incoherencia entre lo que se plantea como formación ciudadana y el tipo de evaluación que se práctica tanto a nivel de aula como a nivel nacional, pues existe una tendencia a cuantificar un contenido que es esencial para los procesos sociales, en vez de cualificarlo y enseñarlo para su aplicación en el diario vivir.

\section{CONCLUSIONES, LIMITANTES Y PROYECCIONES}

El problema que da origen a este estudio está vinculado a la evaluación de un eje temático muy particular, como lo es la formación ciudadana, el que se caracteriza por ser un conocimiento vivido, esencial para el desarrollo de la sociedad. Tras los análisis realizados a las diferentes fuentes de información y de la discusión de estos han surgido las siguientes conclusiones, las cuales pretenden aportar al conocimiento de la evaluación en lo que se refiere a la ciudadanía.

Los instrumentos de evaluación diseñados por el grupo de docentes participantes de esta investigación y los ítems de la prueba SIMCE colocan en evidencia que los contenidos evaluados preferentemente son aquellos vinculados con la dimensión político-jurídica de la ciudadanía, con el foco en estos por sobre las otras dimensiones que componen este eje temático. La evaluación de la ciudadanía revela que la enseñanza de esta, entendiéndose que lo evaluado es lo enseñado, se encuentra anclado en la educación cívica, caracterizada porque sus énfasis están puestos en la enseñanza de la institucionalidad y en los conocimientos conceptuales, sin evidencia de un tránsito hacia un enfoque de formación ciudadana como fue planteado en la reforma educacional en la década de los noventa. Los instrumentos analizados son esencialmente pruebas escritas de tipo combinadas, en las cuales existe una preponderancia de las preguntas objetivas por sobre otro tipo de preguntas. No se evidencia en los instrumentos de evaluación que estos promuevan habilidades como la reflexión y el autoaprendizaje de los estudiantes, dado que nos encontramos con ítems construidos en base a la reproducción de información.

La racionalidad curricular que subyace a la evaluación del eje temático de formación ciudadana es la que se ha denominado racionalidad por objetivos, en la cual el currículum es definido por el Estado, restando con ello la posibilidad a los docentes de actuar como una palanca de transformación social, dado que deben ceñirse a las pautas emanadas del MINEDUC en lo relativo a la enseñanza y a la evaluación. En las evaluaciones analizadas se evidencia que estas promueven el desarrollo de habilidades básicas de aprendizaje, pues la mayoría de los ítems analizados están construidos en base a la medición de objetivos tales como identificar, describir y relacionar, los cuales se encuentran en la taxonomía de Bloom en las primeras habilidades de aprendizaje. De este modo no se evidencian evaluaciones que propicien la extrapolación de contenidos ciudadanos a la vida cotidiana de los estudiantes, ni que coloquen en práctica lo aprehendido en las aulas escolares. 
Por su parte, el modelo de evaluación que subyace a los instrumentos analizados es un modelo basado en estándares, el que se caracteriza por ser considerado más cercano a la objetividad que la evaluación comprensiva, el que se tensiona con la ciudadanía como un conocimiento vivido.

Para finalizar resulta relevante señalar que una de las limitantes de este estudio está basado en que solo se trabajó con los diseños de las evaluaciones de los docentes participantes, así también con un grupo reducido de ítems liberados de la prueba SIMCE, por lo que el análisis y las conclusiones de este estudio solo están referidas a una parte de la evaluación. En lo que se refiere a las proyecciones se espera que este sea el inicio de otros estudios que permitan develar preguntas sobre la evaluación de la ciudadanía en Chile, su enseñanza e impacto en la formación de los estudiantes chilenos.

\section{REFERENCIAS BIBLIOGRÁFICAS}

Agencia de la Calidad de la Educación. (2015). ¿Qué es el SIMCE? Autor. Recuperado desde http:// www.agenciaeducacion.cl/simce/que-es-el-simce/

Ander-Egg, E. (1992). Técnicas de Investigación Social. Buenos Aires: Editorial Magisterio del Río de la Plata.

Andrade, M., \& Miranda, C. (2000). El concepto de ciudadanía en educación. Santiago de Chile: Pontificia Universidad Católica de Chile. Recuperado desde http://200.10.23.169/documentos/ documentos\%20originales/nuevos_sentidos_concepto_ciudadania_educacion.pdf

Aristóteles. (2010). Política. Madrid: Ediciones Escolares S.L.

Beck, U. (1998). ¿Qué es la globalización? Barcelona: Paidós.

Bourdieu, P. (1998). La distinción. Madrid: Taurus.

Calderón, F. (Coord.). (2007). Ciudadanía y Desarrollo Humano: Cuaderno de gobernabilidad democrática 1. Buenos Aires: Siglo XXI Editores Argentina.

Castells, M. (1974). La cuestión urbana. Ciudad de México: Siglo XXI.

Castells, M. (1996). La era de la información. Economía, sociedad y cultura. Madrid: Alianza.

Cheresky, I. (2010). Ciudadanos y política en los albores del siglo XXI. Buenos Aires: Ediciones Manantial.

Comisión Formación Ciudadana. (2004). Informe Comisión formación ciudadana. Santiago: Serie Bicentenario.

Contreras, G. (2004). Prácticas y Concepciones de Evaluación del Aprendizaje en Profesores de Física: Un estudio sobre las prácticas de evaluación y sus fundamentos en profesores de la asignatura de Física en la V región (Tesis de Doctoral). Pontificia Universidad Católica de Chile, Santiago.

Cortina, A. (2003). Hacia un concepto de ciudadanía para el siglo XXI. Revista Misión Joven, 314, $17-24$.

Cox, C., Jaramillo, J., \& Reimers, F. (2005). Educar para la Ciudadanía y la Democracia en las Américas. Washington: Banco Interamericano de Desarrollo.

Donoso, S. (2005). Reforma y Política educacional en Chile 1990-2004: el neoliberalismo en crisis. Revista Estudios Pedagógicos, 31(1), 113-135.

Elbaz, M., \& Helley, D. (2002). Globalización, ciudadanía y multiculturalismo. Granada: Maristán.

Falk, R. (2002). La globalización depredadora. Una crítica. Madrid: Siglo XXI.

Fernández, R. (2013). Politizar el consumo, recuperar la ciudadanía. Barcelona: Alba Sud.

García Canclini, N. (1991). Consumidores y ciudadanos: conflictos multiculturales de la globalización. Ciudad de México: Grijalbo.

García-Huidobro, J. (1999). La Reforma educacional chilena. Madrid: Editorial Popular. 
Estudios Pedagógicos XLIII, Nº 2: 253-276, 2017

EVALUACIÓN DE LOS APRENDIZAJES SOBRE CIUDADANÍA: META EVALUACIÓN DE LOS INSTRUMENTOS

UTILIZADOS EN EL SEGUNDO CICLO BÁSICO CHILENO

Góngora, M. (1981). Ensayo Histórico sobre la Noción de Estado en Chile en los siglos XIX y XX. Santiago: Ediciones La ciudad.

Habermas, J. (1998). Más allá del Estado nacional. Madrid: Trotta.

Himmel, E., Olivares, M., \& Zabalza, J. (1999). Hacia una evaluación educativa. Santiago: Ministerio de Educación.

Kymlicka, W., \& Norman, W. (1997). El retorno del ciudadano. Una revisión de la producción reciente en teoría de la ciudadanía. Revista Ágora, 7, 5-42.

Lozano, M. (2012). Filosofía y Ciudadanía. Bachillerato 1. Madrid: Editorial Mc Graw-Hill.

Maldonado, M. (2003). La privatización de la Educación en Chile. Costa Rica: Internacional de la educación. Oficina regional para América Latina.

Ministerio de Educación. (2012). Bases curriculares de la Educación básica. Historia, Geografía y Ciencias Sociales, año 2012. Autor. Recuperado desde http://www.curriculumenlineamineduc. cl/605/w3-article-14598.html

Ministerio de Educación. (2013). Guiones didácticos para Séptimo básico. Orientaciones para fortalecer la Formación Ciudadana. Autor. Recuperado desde http://portales.mineduc.cl/index2. php?id_portal=18\&id_seccion=4636\&id_contenido $=24563$

O’Donnell, G. (2006). Hacia un Estado de y para la democracia. Contribuciones para el debate. PNUD, 2, 25-64.

OCDE. (2015). Estudio económico de la OCDE para Chile. Autor. Recuperado desde http:// centroestudios.mineduc.cl/tp_enlaces/portales/tp5996f8b7cm96/uploadImg/File/Evidencias/ Evidencias\%20final_noviembre_2015.pdf

Piñuel, J. (2002). Epistemología, metodología y técnicas del análisis de contenido. Galicia: Estudios de Sociolingüística.

Platón. (2009). Diálogos. Ciudad de México: Porrúa.

Platón. (2010). La Republica. Madrid: EDIMAT Libros.

Prieto, M., \& Contreras, G. (2008). Las concepciones que orientan las prácticas evaluativas de los profesores: un problema a develar. Revista Estudios Pedagógicos, 34(2), 245-262.

Ramírez, A. (2010). La evaluación como herramienta para mejorar los procesos educativos de poblaciones socialmente vulnerables. Revista Iberoamericana de Educación, 53(5), 1-10. Recuperado desde http://www.rieoei.org/deloslectores/3778Ramirez.p_

Stake, R. (2006). Evaluación comprensiva y evaluación basada en estándares. Barcelona: Editorial GRAÓ.

Stenhouse, L. (2003). Investigación y desarrollo del currículum. Madrid: Ediciones Morata. 